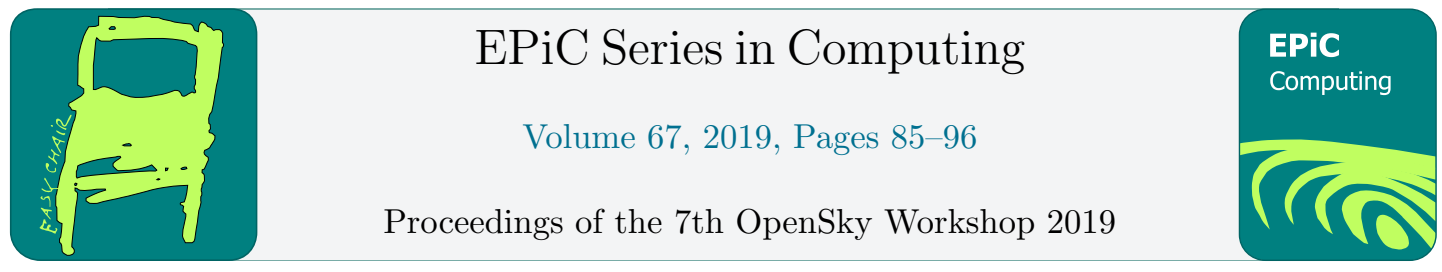

\title{
Jamming/Garbling Assessment and Possible Mitigations in the OpenSky Network
}

\author{
Mauro Leonardi ${ }^{1}$, Martin Strohmeier ${ }^{2}$, and Vincent Lenders ${ }^{2}$ \\ 1 Tor Vergata University, Rome, Italy \\ Mauro.Leonardi@uniroma2.it \\ 2 Armasuisse, Switzerland \\ Martin.Strohmeier@armasuisse.ch,Vincent.Lenders@armasuisse.ch
}

\begin{abstract}
The Automatic Dependent Surveillance-Broadcast (ADS-B) technology is one of the pillars of the future surveillance system for air traffic control. However, its many fundamental vulnerabilities are well known and an active area of research. This paper examines two closely related ADS-B radio frequency channel issues, jamming and garbling.

Both jamming and garbling produce the same physical effect: the reception of mixed signals, coming from different sources (usually not co-located). In this paper, we assess the impact of these reception problems and examine three separate mitigation techniques. Through the use of theoretical evaluations, simulations and real-world analysis based on data collected by the OpenSky Network, we compare their effectiveness and establish a first baseline for their use in modern low-cost, crowdsourced ADS-B networks.
\end{abstract}

\section{Introduction}

Automatic Dependent Surveillance-Broadcast is one of the pillars of the future surveillance system for air traffic control (ATC) [1][2]. It is a straightforward system where the on-board calculated position is broadcast to any possible users on a common radio frequency ( $\mathrm{RF}$ ) channel. The ADS-B system uses a data-link protocol, called "1090 Extended Squitter (1090ES)", that is an evolution of the Identification Friend or Foe (IFF) Secondary Surveillance Radar (SSR) protocol: each aircraft periodically transmits messages (called squitters), pulse positionmodulated on the L-band (1090 MHz) with random access to the channel [3] [4].

Nowadays, country-wide networks of ADS-B receivers are deployed and maintained all over the world by ATC service providers. Moreover, in the last years, various global and volunteerbased crowdsourced networks such as the OpenSky Network [5, 6] were deployed, introducing new services with their own specific challenges and opportunities.

Unfortunately, the ADS-B system suffers from various fundamental security vulnerabilities affecting all components of the system, which have been pointed out repeatedly in the literature $[7,8,9,10,11,12]$. The present work focuses on the 1090ES RF channel vulnerability and in particular on the issues of low throughput caused by garbling and jamming.

Jamming is a well-known electronic warfare technique that intentionally inhibits the reception of the legitimate signals on a given frequency exploiting the transmission of high power 
noise in the same RF band of the system under attack. Nowadays, due to the high availability of very low-cost Software Defined Radios (SDRs), it can be very easy to develop and deploy a simple jammer with dangerous effect on any ADS-B receivers, up to the total denial of the reception of ADS-B messages.

The other well-known weakness of the ADS-B system is the garbling effect: due to random access to the RF channel, messages coming from different aeroplanes may be received superimposed. As the receiver is usually not able to decode superimposed messages, it follows that, if the number of aircraft increases beyond a certain threshold, the overall surveillance capability of the system decreases.

It is clear that these two problems, the intentional jamming and the unintentional garbling, produce the same physical effect on the receiver side, that is the reception of mixed signals, coming from different sources (usually not co-located) that can be different aircraft or an aircraft and a jammer.

In the past, the effects of a jammer on an ADS-B receiver were typically evaluated using real ADS-B signals corrupted by a laboratory implementation of an ADS-B jammer [7, 9]. Several works also considered the issue of modelling the garbling problem and also proposed some solutions $[13,14,15,16]$. However, none of the discussed solutions are suitable for crowdsourced networks because they are tailored for professional high-performance receivers and typically use fully-calibrated multi-channel receivers and a high sampling rate of the incoming signal.

In this work, we instead investigate models for the problem assessment and mitigation techniques suitable for low-cost sensor implementations. First of all, realistic jamming and garbling models for sensors and network of sensors are developed. Subsequently, different mitigation approaches are compared in terms of performance and cost using analytic models, simulations and, where possible, real-world data.

The following three different approaches are investigated and compared:

1. Network-oriented: Exploiting redundancy by improving the number and/or the distribution of sensors in a network can help improve performance under jamming or garbling.

2. Sensor-oriented - Coverage Sectorization: Sectorization, by the use of sector antennas and multi-channel receivers, reduces the messages to be received from each receiver channel. Accordingly, the garbling and jamming problem on each channel can be reduced.

3. Sensor-oriented - Signal Processing: Signal processing can be used to un-mix the garbled/jammed signals. Typically this approach is based on signal's "sparsity" and, in particular, it exploits the geometrical distribution of the different signal sources.

All three proposed models will be used to evaluate the jamming/garbling impact on the OpenSky Network.

\section{Garbling Risk Assessment}

Given a standard ADS-B receiver, the classic Poisson model can be used to calculate the probability of reception of one or more ADS-B messages in a given time window:

$$
p^{j}(n)=\frac{\left(\lambda_{j} N t_{j}\right)^{n}}{n !} e^{-\lambda_{j} N t_{j}}
$$


where $p^{j}(n)$ is the probability that $n$ replies are received in the time window of size $t_{j}, \lambda_{j}\left[s^{-1}\right]$ is the frequency of messages generation of the airplane and $N$ is the total number of airplanes in the receiver coverage area.

Now, assuming that an ADS-B message is received at time $t_{0}$, a possible interference window can be defined for each possible types of interfering messages (Mode A/C, Mode S Short, Mode $\mathrm{S}$ Long), the size of these windows are the time intervals where, if another message arrives, there is an overlapping with the ADS-B one. Their sizes are $t_{1}=120+20=140 \mu \mathrm{s}$ for a Mode $\mathrm{A} / \mathrm{C}, t_{2}=120+64=184 \mu \mathrm{s}$, fore a Mode S Short and $t_{3}=120+120=240 \mu \mathrm{s}$ Mode $\mathrm{S}$ long signals.

Using Equation (1), it is possible to calculate the probability of the correct reception of the ADS-B message with up-to $m$ interfering messages of type $j$ :

$$
p_{r}^{j}(m)=\sum_{m=0}^{M} P_{r}^{j}(m) p^{j}(m)
$$

where $P_{r}^{j}(m)$ is the probability to decode an ADS-B message when superimposed with $m$ messages of type $j$. Considering all the possible types of interference, the total probability to decode the ADS-B massage is given by the combination of the corresponding complex events. A typical assumption for $P_{r}^{j}(m)$ can be derived from the RTCA recommendations [17]:

$$
P_{r}^{2,3}(n)=\left\{\begin{array}{cc}
1 & n=0 \\
0 & n>0
\end{array} \quad P_{r}^{1}(n)=\left\{\begin{array}{cc}
1 & n=0 \\
0.89 & n=1 \\
0.64 & n=2 \\
0.52 & n=3 \\
0 & n>3
\end{array}\right.\right.
$$

For this assumption, a simple model for the total probability to decode an ADS-B message in case of garbling can be expressed as follows:

$$
p_{r}=\left[\sum_{n=0}^{3}\left(P_{r}^{1}(n) p^{1}(n)\right)\right] \cdot p^{2}(0) \cdot p^{3}(0)
$$

Usually, however, a surveillance system can tolerate some lost messages. For this case, it is possible to evaluate the probability of correct surveillance $p_{s}$ of an aircraft, recalling that it is possible to accept a surveillance renewal time higher than the broadcast frequency (e.g., $2 \mathrm{~Hz}$ for an airborne position message). In particular, if the required renewal time is set to five seconds, at least one ADS-B message over nine consecutive ones shall be received. Under this assumption, the probability of surveillance is given by the probability of receiving at least one of the nine messages, that is equivalent to the complement to one of the probability that all the messages are not received; calling $q_{r}$ the probability of not being able to receive a message, it follows:

$$
q_{r} \quad=1-p_{r} ; \quad p_{s}=1-\left(q_{r}\right)^{9}
$$

Finally, it must be noticed that these probabilities refer to the probability of receiving the messages in the coverage area without any assumption on their relative power or their Signal to Noise Ratio (SNR). This is a strong simplification because in case of superimposed messages with big differences in their signal power the most common receivers are designed to be able to 
decode the more powerful one [17]. Under this new assumption, and further assuming that the ADS-B message arrives from an aeroplane at a given distance $R$, it can be supposed that all messages able to produce interference shall originate from aeroplanes with a distance shorter than $R+\Delta R$, where $\Delta R$ is used to take into account a minimum difference in the two signal levels. It is possible to calculate the relationship between the distance of the ADS-B aircraft and the pertinent probability of reception of its messages: calling $R_{1}$ and $R_{2}$ the distances of the two aircraft from the station, the received signal powers and the corresponding Signal to Interference Ratio (SIR) will be:

$$
P_{1}=f\left(1 / R_{1}^{2}\right) ; \quad P_{2} \quad f\left(1 / R_{2}^{2}\right) ; \quad S I R=\frac{P_{1}}{P_{2}}=\frac{R_{2}^{2}}{R_{1}^{2}}
$$

If we suppose that we have an harmful interference only for a Signal to Interference Ratio (SIR) below than a given value (for example $6 d B$ ), all the aircraft having a distance given by the following relationship will be considered sending interfering signals:

$$
R_{2}<\sqrt{S I R \cdot R_{1}^{2}}=\sqrt{4 R_{1}^{2}}
$$

Finally, assuming a given density of aircraft for meters square, $d$, we can compute the number of interfering aircraft to be used in Equation (1):

$$
N=d \cdot \pi R_{2}^{2}=d \cdot S I R \cdot\left(\pi R_{1}^{2}\right)
$$

The above-derived models can also be used to evaluate the expected performances in case of more than one receiver, which is the network-oriented mitigation approach.

For example, suppose we have $K$ independent stations in a random positions (far enough from each other that we can assume the pertinent traffic to be independent) receiving the same ADS-B message. In this case, the probability to receive the message (without interference) with at least one station can be computed, again, as the complement to one of the probability that the message is interfered with on all stations:

$$
q_{r}=1-p_{r} ; \quad p_{s, K}^{N e t}=1-q_{r}^{9 \cdot K}
$$

On the other hand, in case of the sectorization approach, having $K$ receivers co-located in the same sensor, the total coverage of the station can be divided in $K$ different sectors. In this case, the probability of reception $p_{r}$ can be still computed with Equations (4) and (5), but now $p^{j}(n)$ will be (assuming a perfect sectorization, i.e., different sectors don't have common traffic):

$$
p^{j}(n)=\frac{\left[\lambda_{j}(N / K) t_{j}\right]^{n}}{n !} e^{-\lambda_{j}(N / K) t_{j}}
$$

and $p_{s}$ can be computed as before.

Finally, it is possible to improve $p_{r}$ improving the probability to decode a message $\left(P_{r}^{j}(m)\right)$ in the various garbling conditions, that is, improving the signal processing. As mentioned before, a typical approach is the use of a multiple channels receiver, omni-directional antennas and some particular signal processing techniques able to separate superimposed replies exploiting the signals sparsity $[18,19,14]$.

Usually, such techniques are able to separate the signals if a minimum part of the first signal is received without interference. In this case, the interfering windows in Equation (1) can be reduced (for example, if we assume that it is sufficient to receive only the preamble with no interference):

$$
p_{k}^{j}(n)=\frac{\left(\lambda_{j} N t_{j}^{\prime}\right)^{n}}{n !} e^{-\lambda_{j} N t_{j}^{\prime}}
$$


where $t_{j}^{\prime}$ are the new sizes of the interfering windows for the different types of interference. These are, respectively, $t_{1}^{\prime}=8+20 \mu s$ for a Mode A/C, $t_{2}=8+64 \mu s$, for a Mode S Short and $t_{3}=8+120 \mu \mathrm{s}$ Mode $\mathrm{S}$ long signals.

Moreover, the maximum processing performance can be achieved with Digital Beam Forming (DBF)[20] or Blind Source Separation (BBS) techniques [21], in which the signals coming from the different receivers (using different antennas) are combined together to obtain the best possible system performances. In this case, the performances are similar to the one obtained with the sector antennas because the algebraic combination of the signals produces virtual beams that can be pointed digitally. Other advantages include a flexibility gain. For example, it is possible to change the beam patterns every time needed, it is possible to introduce null steering or to use super-resolution and compressive sensing.

In Figure 1 a comparison of the expected surveillance performances for the four different approaches (in case of 4 receivers) is reported.

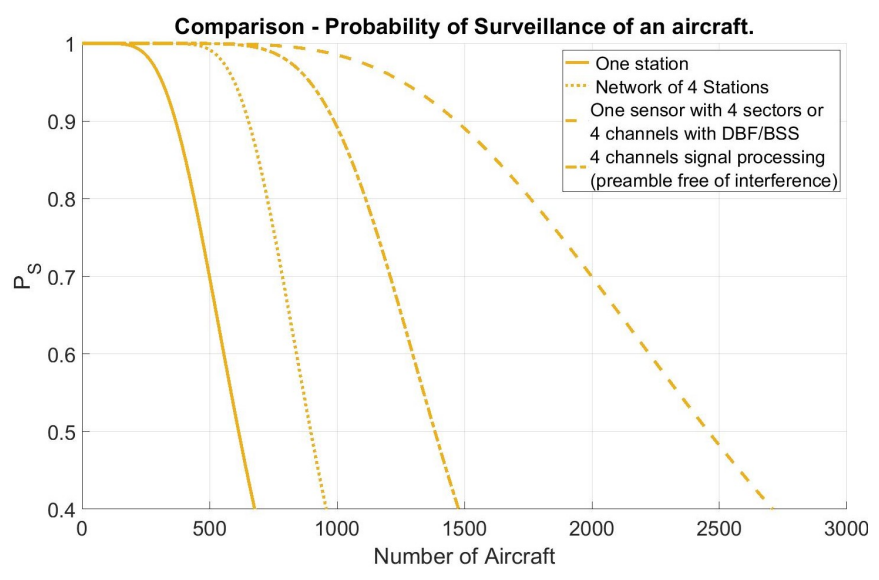

Figure 1: Comparison. Probability of surveillance of an aircraft varying the number of the aircraft $\left(\lambda_{1}=30\right.$ replies $/ \mathrm{sec}, \lambda_{2}=8$ replies $/ \mathrm{sec}$ and $\lambda_{3}=6$ replies $\left./ \mathrm{sec}\right)$.

The figure clearly shows that in case of garbling and fixing the number of receivers, the best performance can be obtained with signal processing or sectorization.

\section{$3 \quad$ Jamming Risk Assessment}

For the assessment of the general jamming risk, we assume a jammer with a given transmitter power $P_{J}$ and a given antenna gain $G_{J}$. Using the Friis equation, it is possible to compute the corresponding received power $P_{J}^{r}$ :

$$
P_{J}^{r}=G_{J} G_{r}\left(\theta_{J}, \phi_{J}\right)\left(\frac{\lambda}{4 \pi R_{J}}\right)^{2} P_{J}
$$

where $R_{J}$ is the jammer-sensor range and $G_{r}\left(\theta_{J}, \phi_{J}\right)$ is the ADS-B antenna gain in the direction of the jammer.

If $P_{J}^{r}$ is higher than the saturation threshold of the receiver, $P_{\text {sat }}$, the reception of any other signals is inhibited. The value of $P_{\text {sat }}$ depends on the receiver hardware and change from receiver to receiver. However, also if the power is lower than the saturation level, the receiver 
performances are reduced. It is possible to compute the received power for a message coming from an ADS-B transponder and than calculate the corresponding SIR:

$$
S I R=\frac{P_{T}^{r}}{P_{J}^{r}}=\frac{G_{T} G_{r}\left(\theta_{T}, \phi_{T}\right) P_{T} R_{J}^{2}}{G_{j} G_{r}\left(\theta_{J}, \phi_{J}\right) P_{j} R_{T}^{2}}
$$

Afterward, imposing a minimum level for this ratio, for example $6 d B$, it is possible to compute the maximum coverage of the receiver in case of jamming:

$$
R_{T}^{2}=\frac{G_{T} G_{r}\left(\theta_{T}, \phi_{T}\right) P_{T} R_{J}^{2}}{4 G_{J} G_{r}\left(\theta_{J}, \phi_{J}\right) P_{J}}
$$

Assuming the same gain of the receiver in the jammer and transponder directions:

$$
R_{T}=\frac{1}{2} \sqrt{\frac{G_{T} P_{T}}{G_{J} P_{J}}} R_{J}=\frac{1}{2} \sqrt{\frac{E I R P_{T}}{E I R P_{J}}} R_{J}
$$

Note that the transponder EIRP (Effective Isotropic Radiated Power) is specified by ICAO [4]: the peak (pulsed) EIRP should be between $125 W(+21 d B W)$ and $500 W(+27 d B W)$ and a lower EIRP limit is further reduced to $70 W(+18.5 d B W)$ for aircraft flying below FL150 (15,000 feet).

In Table 1, we report the maximum coverage for a 500 Watt transponder jammed with 100 $\mathrm{mW}$ of peak power (i.e., for example, the peak power of the common Ettus SDR in L band [22]) and with 20 Watt, respectively, (using a commercial power amplifier) for three different jammer/sensor ranges. It is clear that also with very low power transmitter is possible to totally deny the service of a sensor, if it is placed near the antenna. For larger distances, the effect is reduced and to be harmful the jammer must have an high EIRP (i.e. high transmitter power or a directive antenna).

Table 1: Maximum coverage (and percentage of coverage reduction) in case of a 500 Watt transponder jammed by a jammer with $100 \mathrm{~mW} / 20 \mathrm{~W}$ of peak power for various Jammer/Station ranges.

\begin{tabular}{c|c|c|c|c}
\hline \hline Jammer Range & \multicolumn{2}{|c|}{ EIRP=100 $\mathrm{mW}$} & \multicolumn{2}{c}{ EIRP $=20 \mathrm{~W}$} \\
& Max Range $(\mathrm{Km})$ & Cov.red. (\%) & Max Range $(\mathrm{Km})$ & Cov.red. (\%) \\
\hline 0.5 & $17.68 \mathrm{Km}$ & $98.80 \%$ & $1.25 \mathrm{Km}$ & $99.99 \%$ \\
\hline 1 & $35.36 \mathrm{Km}$ & $99.22 \%$ & $2.5 \mathrm{Km}$ & $99.99 \%$ \\
\hline \hline 10 & $353 \mathrm{Km}$ & $21.87 \%$ & $25 \mathrm{Km}$ & $99.61 \%$ \\
\hline
\end{tabular}

Using sector antennas it is possible to improve the SIR for the signals coming from antennas that do not point in the jammer. A simple evaluation for the case of perfect sectorization can be done assuming that the effect of a jammer will be present only on one sector at a time. In this case, only the coverage of the sector under attack will be reduced as described before. In Table 2 the effects of a jammer on a four sectors sensor are reported. The same assumption (jammer affecting only one virtual beam) can be done for the signal processing techniques based on BSS or Beamforming, for a four channels receiver.

Finally, in case of a network of sensors, finding a simple and general model for the expected performances is difficult because it will depend from the particular geometry between jammer and receivers. In this case, the performance will be evaluated for a given real configuration the OpenSky Network - in the following section. 
Table 2: Maximum coverage in case of a 500 Watt transponder jammed by a jammer with 100 $\mathrm{mW} / 20 \mathrm{~W}$ of peak power. The coverage reduction is obtained considering a nominal range of $400 \mathrm{Km}$. Four Sector Antenna.

\begin{tabular}{c|c|c}
\hline \hline Jammer Range $(\mathrm{Km})$ & EIRP=100 mW. Cov.red. (\%) & EIRP =20 W. Cov.red. (\%) \\
\hline \hline 0.5 & $24.95 \%$ & $24.99 \%$ \\
\hline 1 & $24.80 \%$ & $24.99 \%$ \\
\hline 10 & $5.4688 \%$ & $24.90 \%$ \\
\hline \hline
\end{tabular}

\section{Network Improvements: Trials and Evaluation with OpenSky Data}

In this section, the jamming and garbling mitigation performances are evaluated for a real-world crowdsourced ADS-B network using data provided by the OpenSky Network [23].

Concerning jamming, two different hypothesis are evaluated: a low cost, low power jammer placed on the ground near a receiving station, and a high power jammer flying in the OpenSky coverage area. In the first case, as described in the previous section, the jammer is able to fully deny the service of a single station, but the effects on more distant stations are negligible due to a lack of direct line of sight communication. Under this assumption, the regions of the OpenSky Network coverage directly vulnerable to the jammer will be those regions in which ADS-B messages are received with only one sensor.

In Figure 2 this part of OpenSky Network coverage is reported. The area, shown in blue, makes up about $49 \%$ of the total coverage. In these regions, a single jammer can thus directly cause a loss of coverage of thousands of square kilometers.

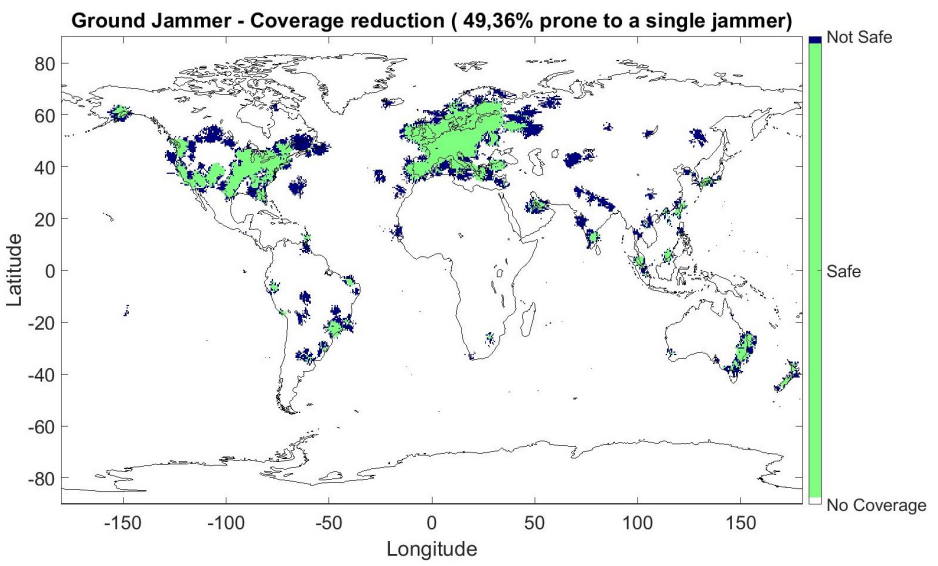

Figure 2: Simple single ground jammer unsafe region (24 August 2019).

In the case of a mobile flying jammer, the distances between jammer and receivers are relatively large, and the coverage reduction effect is reduced but, in this case, the jammer affects more than one station at a time. To evaluate this, simulations with a flying jammer in the Europe sky were done. For each jammer position, the jammer-reduced coverage is computed for all the stations in the view and the new OpenSky overall coverage computed. 

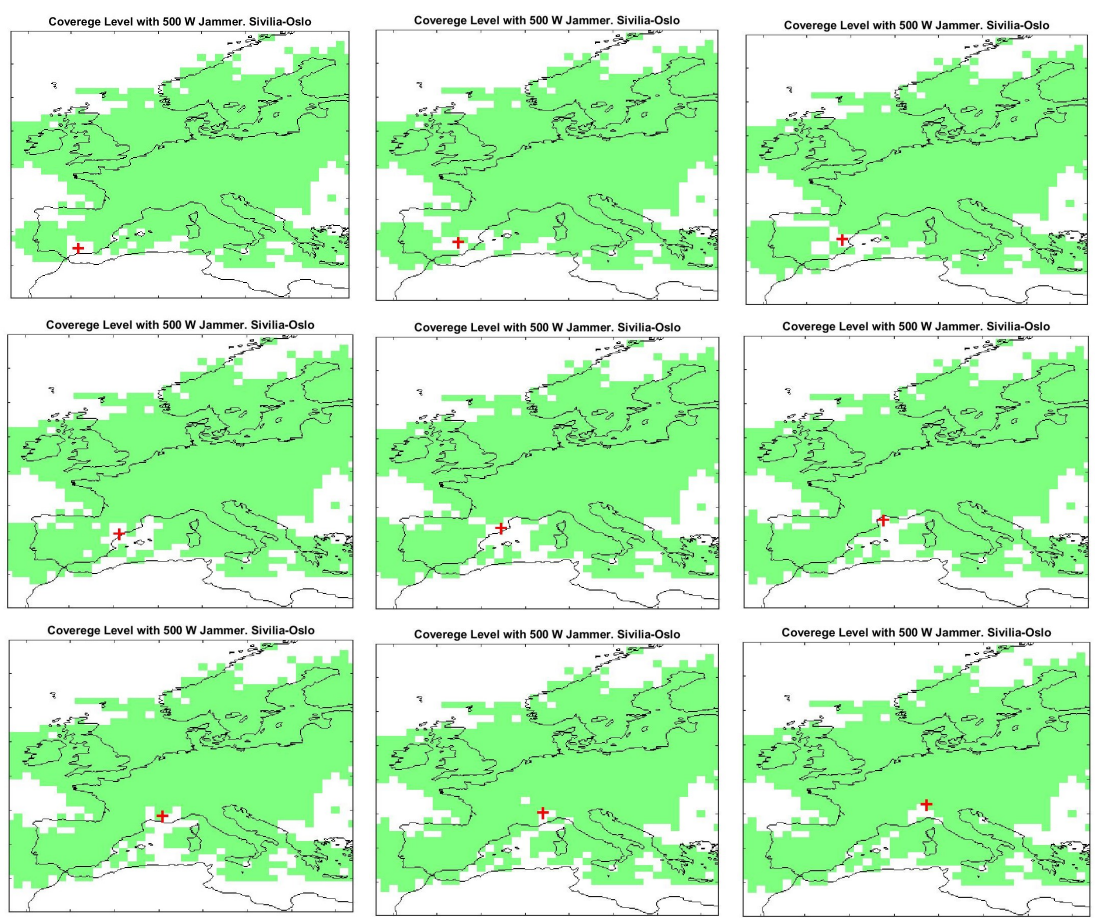

Figure 3: Effect of a Flying jammer moving from Seville to Frankfurt on the OpenSky Network (24 August 2019). Jammer power equal to $500 \mathrm{~W}$.

In Figure 3 an example of the total coverage (green zone) obtained when a 500 Watt jammer flying between Seville and Frankfurt is reported. It is possible to see that the effect of the jammer is mitigated when it is near Frankfurt due the high number of stations in the view.

Concerning the garbling problem, a possible evaluation of its impact on the OpenSky Network can be done using the state vectors provided by the network. These reports are carried out every second for each aircraft under surveillance, providing information such as aircraft position and velocity but also the time of last contact (i.e., the time stamp of the last received message from the aircraft). As shown in Equation (3) the probability to receive at least a reply from an aircraft in the last $S$ seconds in a network of receiver can be easily related to the number of the sensors $(M)$, the number of replies for aircraft $(\lambda)$, the number of aircraft $(N)$ and the receivers performances $\left(P_{r}^{j}\right)$. Assuming that the performance, the geometry and the number of the receivers for a given region do not change over time and, assuming also that the aircraft reply emission rate does not change, the surveillance probability will depend only on $N$, or rather on the probability to have a message conflict (Equation 1), in which only $N$ can change due to the changing of the traffic conditions. It follows that computing the ratio between the number of reports with the last contact timestamp older than $S$ and the total number of reports, will give the impact of the garbling effect on a given region.

In Figure 4 examples of the ratio between reports with an "age" higher than 8 seconds over the total number of reports are shown, for four different regions in Europe.

Two effects can be highlighted. First of all, the average performance level is different for different regions. This effect is related to the different geometries, different number of sensors, and different sensor performance. The second effect is of a time-dependent nature. This periodic 


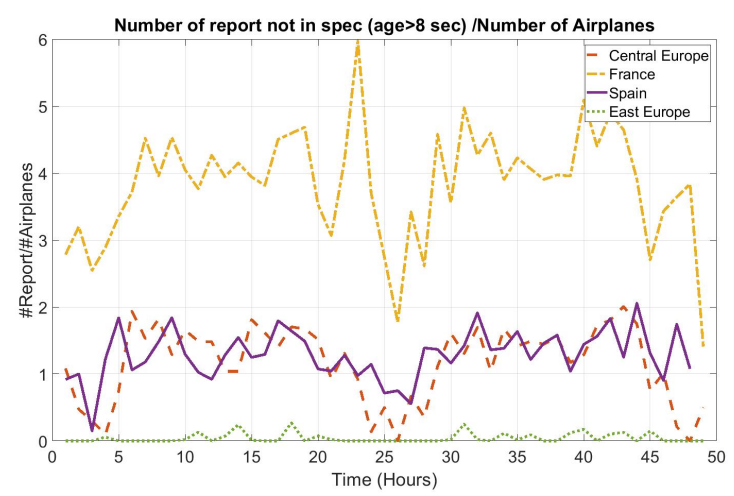

Figure 4: Comparison of the garbling effects in different Europe regions.

behaviour is related to the change of the number of flights over time; the fewest reports can be observed during the night hours when the number of flights is low. Since all the other parameters are fixed, this value changing in time can be assumed as related to the garbling effect. The effect correlates positively with the number of aircraft in the coverage area.

Finally, a reduction of the surveillance probability is observed for all tested areas; it follows that a solution based only on high redundancy cannot be used to totally solve the garbling problem, confirming the evaluation done in the previous section.

\section{Station improvements: First results}

As mentioned in the introduction, the performance of a single station can be improved using co-located receivers and BSS. In recent works [24, 25], the authors use simulations to show that Blind Source Separation based on Principal Component Analysis can be used for garbling and jamming reduction in Mode $\mathrm{S}$ signals even in the case of low-cost receivers.

The design, implementation and evaluation of simplified algorithms suitable for low-cost hardware are ongoing, and large-scale trials with real data will be done in future work. However, a first example of degarbling using real signal received with low-cost hardware is reported here.

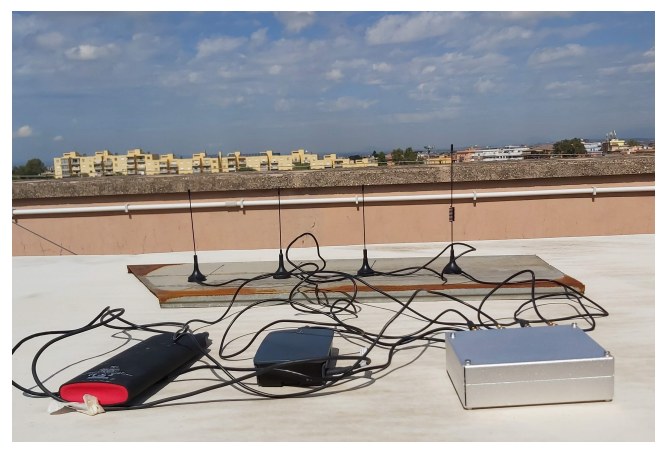

Figure 5: Kerberos-RTL and cheap antennas configuration.

A Kerberos-SDR receiver [26] (together with a Raspberry Pi and 4 low-cost antennas) was 
used to record live Mode S signals (see Figure 5). The recorded signals were then decoded on each channel using an algorithm similar to the one used in dump1090 [27]. If a preamble was found in at least one channel but no replies were decoded, the degarbling method was applied to separate the (possibly) garbled signals and a new decoding phase was attempted on each principal component. The effect of this processing can be seen in Figure 6 where a preamble is detected on the received signals (Figure 6 - bottom), but no reply was decoded without errors. After PCA processing, the first two principal components clearly show a Mode S long message and a Mode A/C message (Figure 6 - top). Moreover, analysing the first results, an improvement of the number of low-SNR/not-garbled replies decoded without errors (thanks to the signal processing gain) was observed.
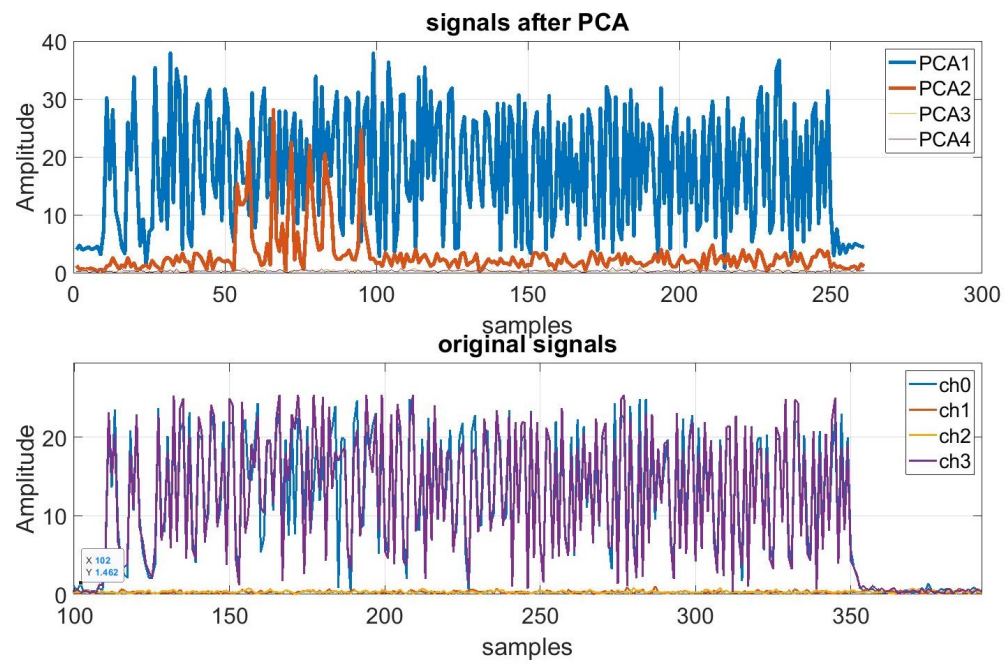

Figure 6: Example of de-garbling. Real data received with a Kerberos-RTL.

\section{Conclusion}

Several approaches to increase the overall performance of a network of ADS-B receivers in term of robustness with respect to jamming and garbling were evaluated. In particular, three different solutions were analysed: improving the number of sensors in the coverage area or improving the single sensor performances by the use of multi-channels receiver and sector antennas or multi-channels with omni-directional antennas and signal processing. A qualitative comparison of the possible improvements and costs is reported in Table 3 (summing-up theoretical analyses, simulations and real data analyses). It must be noted that these preliminary results do not show a clear winner, each has strengths and weaknesses. In general, the best solutions should consider the overall coverage requirements and the existing sensor distributions of the target receiver network. The discussed single receiver solutions will be designed to be compatible and available for low-cost sensors, in hope of wide adaptation by the ADS-B sensor operators. This should improve the overall performance of the network of sensors, in particular for the case of organically growing non-controlled sets of receivers such as the OpenSky Network. 
Table 3: Preliminary comparison of the different approaches. Costs and expected performances.

\begin{tabular}{r|c|c|c}
\hline \hline & Network & Sectorization & Signal Processing \\
\hline Receiver Cost & $*$ & $* * *$ & $* *$ \\
Antenna Cost & $*$ & $* *$ & $* *$ \\
\hline Back Bone Cost & $* *$ & $* * *$ & $* *$ \\
Garbling mitigation & $*$ & $* *$ & $* *$ \\
Jamming mitigation (Ground attack) & $* * *$ & $* * *$ & $* * *$ \\
\hline Other & none & SNR improvement & $\begin{array}{c}\text { SNR improvement } \\
\text { (processing gain) }\end{array}$ \\
\hline \hline
\end{tabular}

(*: Low; ${ }^{* *}$ : Medium; ${ }^{* * *}$ : High.)

\section{Acknowledgments}

The authors gratefully acknowledge the financial support of Armasuisse Science \& Technology.

\section{References}

[1] NEXTGEN. https://www.faa.gov/nextgen/.

[2] SESAR. http://www.sesarju.eu/.

[3] M.C. Stevens. Secondary Surveillance Radar. Artech House, 1988.

[4] ICAO. Annex 10 to the Convention on International Civil Aviation Aeronautical Telecommunication, 1998.

[5] https://opensky-network.org/.

[6] Matthias Schäfer, Martin Strohmeier, Vincent Lenders, Ivan Martinovic, and Matthias Wilhelm. Bringing up opensky: A large-scale ads-b sensor network for research. In Proceedings of the 13th international symposium on Information processing in sensor networks, pages 83-94. IEEE Press, 2014.

[7] M. Leonardi, E. Piracci, and G. Galati. Ads-b vulnerability to low cost jammers: Risk assessment and possible solutions. In Digital Communications - Enhanced Surveillance of Aircraft and Vehicles (TIWDC/ESAV), 2014 Tyrrhenian International Workshop on,, pages 41-46, Rome, 2014.

[8] M. Strohmeier, V. Lenders, and I. Martinovic. On the security of the automatic dependent surveillance-broadcast protocol. IEEE Communications Surveys \& Tutorials, 17(2):1066-1087, 2015.

[9] Leonardi, Piracci, and Galati. Ads-b jamming mitigation: a solution based on a multichannel receiver,. IEEE Aerospace and Electronic Systems Magazine, 32(11):44-51, 112017.

[10] Mauro Leonardi, Luca Di Gregorio, and Davide Di Fausto. Air traffic security: Aircraft classification using ads-b message's phase-pattern. Aerospace, 102017.

[11] J. Butts, D. McCallie, and R. Mills. Security analysis of the ads-b implementation in the next generation air transportation system. International Journal of Critical Infrastructure Protection, 4(2):78-87, 082011.

[12] K. Sampigethaya and R. Poovendran. Visualization \& assessment of ads-b security for green atm. In AIAA/IEEE Digital Avionics Systems Conference - Proceedings, 2010.

[13] G. Di Massa, S. Costanzo, A. Borgia, I. Venneri, G. Galati, M. Leonardi, and E. Piracci. Multiple sources discrimination by array processing. In Proceedings of the 5th European Conference on Antennas and Propagation (EUCAP), pages 620-622, April 2011. 
[14] N. Petrochilos, G. Galati, and E. Piracci. Separation of ssr signals by array processing in multilateration systems. IEEE Transactions on Aerospace and Electronic Systems, 45(3):965-982, July 2009.

[15] N. Petrochilos, G. Galati, and E.G. Piracci. Separation of ssr signals by array processing in multilateration systems. IEEE Transactions on Aerospace and Electronic Systems, 45(3):965-982,, 2009.

[16] G. Galati and M. Leonardi. Super-resolution processor/receiver to discriminate superimposed secondary surveillance radar (ssr) replies and squitter. patent no us6819282 b1, 2004.

[17] RTCA Inc. Minimum Operational Performance Standards for $1090 \mathrm{MHz}$ Extended Squitter Automatic Dependent Surveillance - Broadcast (ADS-B) and Traffic Information Services Broadcast (TIS-B). DO-260B with Corrigendum 1, December 2011.

[18] N. Petrochilos, G. Galati, and E. Piracci. Projection techniques for separation of multiple secondary surveillance radar sources in a real environment. In Fourth IEEE Workshop on Sensor Array and Multichannel Processing, 2006., pages 344-348, July 2006.

[19] E. G. Piracci, N. Petrochilos, and G. Galati. Mixed ssr sources separation exploiting sparsity: a geometrical approach. In 2009 European Radar Conference (EuRAD), pages 85-88, Sep. 2009.

[20] John Litva and Titus K. Lo. Digital Beamforming in Wireless Communications. Artech House, Inc., Norwood, MA, USA, 1st edition, 1996.

[21] Naik Ganesh and Wang Wenwu. Blind Source Separation Advances in Theory, Algorithms and Applications. Springer, 2014.

[22] ETTUS. https://www.ettus.com/product/details/un210-kit.

[23] https://opensky-network.org/contribute/improve-coverage.

[24] M. Leonardi and E. Giuseppe Piracci. Ads-b degarbling and jamming mitigation by the use of blind source separation. In 2018 IEEE/AIAA 37th Digital Avionics Systems Conference (DASC), pages 1-5, Sep. 2018.

[25] M. Leonardi and M. Maisano. Degarbling technique for low cost ads-b receivers. pages 65-69, 2019. cited By 0.

[26] INDIEGOGO. https://www.indiegogo.com/projects/kerberossdr-4x-coherent-rtl-sdr/, November 2018.

[27] https://github.com/antirez/dump1090. 